\title{
Risks and Benefits Associated with Tribulus terrestris Products Assessed by Phytochemical and Pharmacological Screening
}

\author{
RUXANDRA STEFANESCU ${ }^{1}$, ESZTER LACZKO-ZOLD ${ }^{1 *}$, ANCA MARE ${ }^{1}$, \\ SIGRID ESIANU ${ }^{1}$, IZABELA GRAMA ${ }^{2}$, ANCUTA NEGROIU ${ }^{1}$, CAMIL VARI ${ }^{1}$ \\ ${ }^{1}$ George Emil Palade University of Medicine, Pharmacy, Science, and Technology of Targu Mureș, Faculty of Pharmacy, Targu Mureș, \\ Romania \\ ${ }^{2}$ Wessling, Targu Mureș, 10 Paul Chinezu Str., 540326, Targu Mures, Romania
}

\begin{abstract}
Recommendations of products with Tribulus terrestris as anabolic enhancers are based on empirical data. Exposure, especially of athletes, is very high, being among the most widely used nutritional supplements. Consumption of products containing Tribulus terrestris extracts has increased rapidly in the past few years due to the high content of sterol saponosides, compounds with presumed anabolic properties. Because of this upward surge, their efficacy and/or their safety are more and more often questioned. Commercialized dietary supplements, are often mislabelled, adulterated, or contaminated, therefore an evaluation of benefit/risk balance should always be conducted. Our study aimed to analyse an herbal drug and a food supplement frequently encountered on the market. Total polyphenolic and total saponosides content were determined. Inductively coupled plasma atomic emission spectrometry (ICP-AES) was used to determine mineral element content of Tribulus terrestris products. Antioxidant and antimicrobial activities were assayed. The extract obtained with the herbal drug had no antibacterial effect. The extract from the capsules, however, was active against all tested strains with higher activity against Methicillin-resistant Staphylococcus aureus and Escherichia coli $($ MIC $=0.78 \mathrm{mg} / \mathrm{mL})$. Trace elements were quantified, and dose-related risks were outlined, raising awareness regarding the aluminum and nickel content in the herbal drug.
\end{abstract}

Keywords: steroidal saponins, anabolic enhancer, food supplements

\section{Introduction}

Tribulus terrestris (Zygophyllaceae family), also known as puncture vine, is a perennial plant that grows mainly in arid regions. The plant has been used in traditional Indian and Chinese medicine for the treatment of urinary, gastrointestinal, and cardiovascular ailments. Nevertheless, its renown is due to its presumed tonic, anabolic, and aphrodisiac effects [1, 2]. Some of these pharmacological properties have been tested in vitro and in vivo in recent decades. The aphrodisiac activity was carried out on male rats and is probably due to its androgen increasing effect $[3,4,5]$. This wide range of hormone-related effects stimulated the production and consumption of food supplements that contain TT fruit extracts. Steroidal saponins are thought to modulate reproductive metabolism; therefore, these products are used as a replacement for anabolic steroids. Of the steroidal saponins protodioscin, a bisglycoside form of diosgenin, is the major compound. Other steroidal saponins of furostanol and spirostanol type have been identified: prototribestin, protogracillin, etc. [6, 7]. Alkaloids and flavonoids have also been identified in the extracts obtained from fruits; therefore, the extracts are considered to also have antioxidant effects (attributed mainly to di-p-coumaroylquinic acid derivatives) $[8,9]$.

Due to their composition, herbal supplements containing Tribulus terrestris are frequently used by athletes. Because of this upward surge, their efficacy and their safety are more and more often questioned. The objectives of this study were to phytochemically and pharmacologically characterize a Tribulus terrestris herbal drug and a food supplement easily accessible on the Romanian market.

*email: eszter.laczko@umfst.ro; zoldeszter@yahoo.com 


\section{Materials and methods}

\subsection{Sample and extract preparation}

The herbal drug abbreviated TT-HD (Tribulus terrestris fruits, originated from China, organic certified) was purchased from a local pharmacy and the sample was authenticated at the Department of Pharmacognosy and Phytotherapy, based on its macro- and microscopic characteristics. A voucher specimen has been kept at the Department of Pharmacognosy, Faculty of Pharmacy, George Emil Palade University of Medicine, Pharmacy, Science and Technology from Târgu Mureș, Romania. (RSTT-171). The food supplement (in the pharmaceutical form of capsules) abbreviated TT-FS was ordered from an online store, and its label stated that it contained a lyophilized extract with a $60 \%$ content of saponins. No other information regarding the composition or the methods of extraction was given. The accurately weighted content from the capsules was suspended in water and placed in an ultrasound water bath for $30 \mathrm{~min}$. The herbal drug was extracted under the same conditions using ultrasound-assisted extraction.

\subsection{Chemicals and instrumentation}

Spectrophotometric determinations were performed on a UV-VIS spectrophotometer (Specord 210, Analytic Jena). For the total phenolic content, Folin-Ciocalteu phenol reagent (Scharlau, Spain), sodium carbonate (Alfa Aesar, Karlsruhe, Germany), and gallic acid (Sigma) were used. For the DPPH radical scavenging activity 1,1-Diphenyl-2-picrylhydrazyl (DPPH) and ascorbic acid from Sigma were used. For the ABTS radical scavenging activity, 2,2'-azino-bis (3-ethylbenzothiazoline) -6 sulfonic acid diammonium salt, potassium persulfate and Trolox were obtained from Sigma.

The solvents used for extraction were purchased from Merck (Darmstadt, Germany). Water was double distilled and purified with the Direct-Q system (Millipore, Bedford, USA).

\subsection{Total polyphenolic content (TPC)}

Total polyphenolic content was determined using a modified method described by Singleton and Rossi [10]. Briefly, $40 \mu \mathrm{L}$ of the sample was mixed with $200 \mu \mathrm{L}$ Folin-Ciocalteu reagent and $20 \%$ sodium carbonate. The mixture was allowed to stand in darkness for $30 \mathrm{~min}$ and the absorbance was read at $765 \mathrm{~nm}$. TPC was determined from the linear equation of a standard curve prepared with gallic acid. The results were expressed as $\mathrm{mg}$ gallic acid equivalents/ $100 \mathrm{~g}$ herbal drug or dietary supplement.

\subsection{Total saponin content (TSC)}

Total saponins from the herbal drug and the extract from the capsules were determined using a modified gravimetric method [11]. Briefly, the herbal drug and the content from the capsules were accurately weighted and repeatedly extracted with $20 \%$ ethanol at $55^{\circ} \mathrm{C}$ for 40 minutes in an ultrasonic water bath. The reunited solutions were then introduced in a separating funnel with $40 \mathrm{~mL}$ diethyl ether and shaken vigorously. After the repeated extraction with diethyl ether, the hydro-alcoholic layer was extracted with a total volume of $120 \mathrm{~mL} n$-butanol. The organic phase was washed with 5\% sodium chloride solution and evaporated to dryness. Further drying was performed in an oven until constant weight. TSC was calculated with the formula: (weight of the residue/ weight of sample x 100).

\subsection{Antioxidant activity}

The scavenging activity of the samples was determined with a spectrophotometric method, using 2,2-diphenyl-1-picrylhydrazyl radical (DPPH•). In a DPPH solution mixed with an extract that can donate a hydrogen atom, the DPPH radical will be transformed in its reduced form, the transformation that can be observed in the change in color from violet to light yellow. Briefly, $500 \mu \mathrm{L}$ sample (six different concentrations) was mixed with $2500 \mu \mathrm{L}$ DPPH 0.1 solution. The mixtures were allowed to stand for $30 \mathrm{~min}$ and then the absorbance was read at $517 \mathrm{~nm}$ [12].

The extract from the capsules was weighed and suspended in water, and the determination was conducted as described above. 
The concentration of the sample required to scavenge $50 \%$ of the DPPH free radical $\left(\mathrm{IC}_{50}\right)$ was determined using the percentage inhibitions plotted using a logarithmic $\mathrm{x}$-axis (concentration).

Inhibition $\%=[(\mathrm{A}$ control $-\mathrm{A}$ sample $) / \mathrm{A}$ control $] * 100$

The antioxidant activity was also evaluated with the ABTS method described by Re et al. with slight modifications [13]. ABTS radical cation $\left(\right.$ ABTS $^{\bullet+}$ ) was produced by the reaction of $7 \mathrm{mM}$ ABTS solution with $2.45 \mathrm{mM}$ potassium persulfate during $12-16 \mathrm{~h}$ at room temperature in the dark. The ABTS $^{\bullet+}$ solution was diluted with methanol to an absorbance of $0.70( \pm 0.02)$ at $734 \mathrm{~nm}$. Trolox was used as an antioxidant standard. After addition of 10-100 $\mu \mathrm{L}$ of sample or Trolox standard to $2.5 \mathrm{~mL}$ of diluted ABTS $^{\bullet+}$ solution, absorbance at $734 \mathrm{~nm}$ was measured at $6 \mathrm{~min}$.

\subsection{Inductively coupled plasma optical emission spectroscopy}

Analysis of 15 metals using inductively coupled plasma optical emission spectroscopy (ICP-OES) was performed for the herbal drug and the dietary supplement on Perkin Elmer ICP-OES Optima 7300 DV. The accurately weighed herbal drug and the content from the dietary supplement were digested with concentrated $\mathrm{HNO}_{3}$ using microwave-assisted digestion. After the digestion, the samples were transferred to a calibrated flask and diluted accordingly with purified water. All results were expressed in $\mathrm{mg} / \mathrm{kg}$ sample (mean).

\subsection{Antibacterial activity}

Antibacterial activity was tested on six different bacterial strains: Staphylococcus aureus ATCC 25923, Methicillin-resistant Staphylococcus aureus (MRSA) ATCC 43300, Enterococcus faecalis ATCC 29212, Escherichia coli ATCC 25922, Klebsiella pneumoniae ATCC 13883, Pseudomonas aeruginosa ATCC 27853. For the determination of minimal inhibitory concentration, the microdilution method was used. Binary dilutions were performed from the tested substances, in 96 wells microdilution plates. $10 \mu \mathrm{l}$ of $0.5 \mathrm{McFarland}$ suspension (prepared from fresh bacterial culture) were mixed with $9990 \mu \mathrm{l}$ of Muller Hinton $2 \mathrm{X}$ broth. From this suspension, $100 \mu 1$ were mixed with the binary dilutions. The plates were incubated at $37^{\circ} \mathrm{C}$ for 18 hours under aerobic conditions. Positive/negative controls were performed for every strain/sample used. The results were expressed as the minimal inhibitory concentration (MIC), as described previously [14].

\subsection{Data analysis}

Statistical analysis was performed using GraphPad Prism 7.0. Data are reported as mean \pm standard deviation (SD) for triplicate determinations. Student $t$-test was used to evaluate statistical differences between the two samples. Differences were considered significant at $\mathrm{p}<0.05$.

\section{Results and discussions}

\subsection{Total polyphenolic content, total saponin content and antioxidant activity}

Herbal product consumption has become a habitual practice in the general population. Online shopping and quick access to information have contributed to this elevated squander. However, the safety issue must be discussed because, with insufficient knowledge, people expose themselves to considerable risks, either by wanting to increase the daily intake in natural compounds with antioxidant activity or by searching for alternatives to allopathic treatments.

We performed a preliminary investigation on the phytochemical composition and pharmacological activity of the Tribulus terrestris herbal drug and a dietary supplement that contains Tribulus terrestris extract.

The results obtained from quantitative determinations had shown that the capsules contain a significant higher concentration of both saponosides and polyphenols. Both samples were tested for their antioxidant activity with two methods and the antimicrobial activity was measured on six different gram-negative and gram-positive microorganisms. Table 1 presents the TPC, TSC and IC50 determined with DPPH and ABTS methods. 
Table 1. Total saponin content (TSC), total polyphenolic content (TPC), and antioxidant activity

\begin{tabular}{|c|c|c|c|c|}
\hline Sample & $\begin{array}{c}\text { TSC } \\
\mathrm{g} / 100 \mathrm{~g}\end{array}$ & $\begin{array}{c}\text { TPC } \\
\text { GAE } \mathrm{mg} / 100 \mathrm{~g}\end{array}$ & $\begin{array}{c}\text { DPPH } \\
\mathrm{IC}_{50}(\mathrm{mg} / \mathrm{mL})\end{array}$ & $\begin{array}{c}\text { ABTS } \\
\mathrm{IC}_{50}(\mathrm{mg} / \mathrm{mL})\end{array}$ \\
\hline TT-HD & $1.9 \pm 0.51$ & $25.1 \pm 0.12$ & $23.33 \pm 3.93$ & $3.47 \pm 0.11$ \\
\hline TT-FS & $18.76 \pm 2.47 *$ & $232.30 \pm 4.6^{*}$ & $0.31 \pm 0.02 *$ & $0.02 \pm 0.001 *$ \\
\hline
\end{tabular}

Ultrasound-assisted extraction is included in the green extraction technologies because of the improved mass transfer through the disruption of the plant cell wall, and, therefore, it usually has a better yield [15].

The content of polyphenols in an extract is frequently influenced by the polarity of the solvent used for extraction. Being unaware of the solvent used in the process of extraction for the dietary supplement makes the interpretation of the results difficult. Due to the chemical characteristics of steroidal glycosides, as they have two parts: a hydrophilic component, the sugar moiety, and a hydrophobic part, the aglycone, the extraction is usually made with water, alcohol or aqueous alcohol. The extraction temperature was chosen according to previously published data which concluded that the temperature used in the extraction process strongly influenced the yield of the extraction $[15,16]$.

The extract contained in the food supplement exhibited the highest antioxidant activity. Oxidative stress occurs when the balance between reactive oxygen species and antioxidants is inclined towards the production of free radicals. Polyphenolic compounds are known to have strong antioxidant properties correlated with their functional groups.

Plant secondary metabolites are known to possess antioxidant activities. Although the concentration of total polyphenols is lower than in other herbal preparations, the antioxidant activity is notable for both products [17]. The advantage of these types of preparations is that the co-occurrence of the saponins in the extract enhances the solubilization of the polyphenolic compounds and the in vivo absorption.

The aforementioned results confirmed that the extracts from both samples exhibited radical scavenging activity. The scavenging activity against DPPH and ABTS radicals by the extract obtained from the herbal drug, expressed as $\mathrm{IC}_{50}$ was similar to the value reported by Dimitrina et al. [9]. The extracts from the capsules possessed the highest antioxidant activity. Findings of Hammoda et al. suggest that the most active constituents from TT with antioxidant effects are coumaroylquinic acids [18]. Antioxidant activity of Tribulus terrestris products is of great importance because of its recommendations as an enhancer in erectile dysfunction. Erectile dysfunction is a multifactorial condition/symptom, but one of the causes includes an accumulation of AGE (advanced glycation endproducts) and reactive oxygen species, compounds that react with nitric oxide [19]. Nitric oxide mediates one of the key mechanisms involved in erection through activation of guanylate-cyclase, which results in an increase of cyclic guanosine monophosphate (cGMP) and dilation of the smooth muscles of the corpus cavernosum.

\subsection{Inductively coupled plasma optical emission spectroscopy}

Inorganic mineral elements contribute, besides organic constituents, to the beneficial effects of plant products. Due to the increased pollution and other environmental factors, investigations of microelement content in the herbal products should be considered a routine screening. An accumulation of toxic trace elements could have a serious negative impact on the population that consumes these products [20].

The content of 5 macroelements $(\mathrm{Na}, \mathrm{K}, \mathrm{Ca}, \mathrm{Mg}$, and $\mathrm{P})$ and ten trace and ultra-elements $(\mathrm{Fe}, \mathrm{Mn}$, $\mathrm{Zn}, \mathrm{Cu}, \mathrm{Co}, \mathrm{Ni}, \mathrm{Pb}, \mathrm{Cd}, \mathrm{Cr}$, and $\mathrm{Al}$ ) in Tribulus terrestris herbal drug and food supplement were 
determined (Table 2).

Table 2. Macro and trace element content of Tribulus terrestris products

\begin{tabular}{ccc}
\hline Minerals & $\begin{array}{c}\text { TT-HD } \\
(\mathrm{mg} / \mathrm{kg})\end{array}$ & $\begin{array}{c}\mathrm{TT}-\mathrm{FS} \\
(\mathrm{mg} / \mathrm{kg})\end{array}$ \\
\hline $\mathrm{Al}$ & 1537.8 & 102 \\
\hline $\mathrm{Ca}$ & 21308 & 471 \\
\hline $\mathrm{Cd}$ & 0.25 & $<\mathrm{DL}$ \\
\hline $\mathrm{Co}$ & 1.36 & $<\mathrm{DL}$ \\
\hline $\mathrm{Cr}$ & 3.8 & 0.056 \\
\hline $\mathrm{Cu}$ & 10.27 & 0.768 \\
\hline $\mathrm{Fe}$ & 2261.8 & 27 \\
\hline $\mathrm{K}$ & 12949 & 7244 \\
\hline $\mathrm{Mg}$ & 4175.7 & 1676 \\
\hline $\mathrm{Mn}$ & 93.67 & 70.3 \\
\hline $\mathrm{Na}$ & 211.56 & 542 \\
\hline $\mathrm{Ni}$ & 4.1245 & 3.16 \\
\hline $\mathrm{P}$ & 1947.7 & 344 \\
\hline $\mathrm{Pb}$ & 2.22 & $<\mathrm{DL}$ \\
\hline $\mathrm{Zn}$ & 23.15 & 10.1 \\
\hline
\end{tabular}

TT-HD - Tribulus terrestris herbal drug

TT-FS - Tribulus terrestris food supplement

$<\mathrm{DL}$ - value under the detection limit

According to the WHO recommendations and assuming a daily intake with $5 \mathrm{~g}$ Tribulus terrestris herbal drug, the contribution of the essential elements to the diet is low except for Fe (over 140\% of RDA) and Mn (20\% of the RDA) [21]. Moreover, fruits of TT had a high content of Al (1537.8 mg/kg TT), which at first glance may seem much. According to the EFSA, in the European population, the estimated daily dietary exposure to aluminium varied from 0.2 to $1.5 \mathrm{mg} / \mathrm{kg} \mathrm{BW} /$ week, in some cases over $2 \mathrm{mg} / \mathrm{kg} \mathrm{BW} /$ week, at $70 \mathrm{~kg} \mathrm{BW}$ this means $140 \mathrm{mg} /$ week [22]. In these parameters, the daily consumption of $5 \mathrm{~g}$ TT herbal drug has a contribution of $53.823 \mathrm{mg} \mathrm{Al} /$ week. If there is a large amount of aluminium intake from food and water, diet supplementation with TT products in the long term requires caution in order to avoid possible toxic effects. The Ni content of the herbal drug is alarming because consumption of $5 \mathrm{~g}$ represents $20 \%$ of the tolerable upper intake level. Recent research has shown that dietary nickel can cause dermatitis. Therefore, sources with high nickel content must be avoided by persons with such skin conditions [23]. Cadmium, cobalt, and lead were under the limit of detection in the food supplement.

\subsection{Antibacterial activity}

Antimicrobial resistance is a severe threat to society and an increasing concern for therapeutic strategies. In recent years, research has been done to isolate natural compounds with potent antibacterial effects against human pathogens.

The results of the antibacterial effects of the TT extract and TT food supplement are presented in Table III. The extract obtained from the herbal drug did not show any antibacterial effect against the 
tested strains. Comparatively, the capsules had an antibacterial effect against all tested strains with a MIC value between 0.78 and $6.25 \mathrm{mg} / \mathrm{mL}$.

Table 3. Antibacterial activity of herbal drug and food supplement

\begin{tabular}{|c|c|c|}
\hline \multirow{2}{*}{ Microorganisms } & \multicolumn{2}{|c|}{ MIC (mg/ml) } \\
\hline & TT-HD & TT-FS \\
\hline \multicolumn{3}{|l|}{ Gram-positive } \\
\hline Staphylococcus aureus & - & 1.56 \\
\hline MRSA & - & 0.78 \\
\hline Enterococcus faecalis & - & 1.56 \\
\hline \multicolumn{3}{|l|}{ Gram-negative } \\
\hline Escherichia coli & - & 6.25 \\
\hline Klebsiella pneumoniae & - & 0.78 \\
\hline Pseudomonas aeruginosa & - & 1.56 \\
\hline
\end{tabular}

TT-HD - Tribulus terrestris herbal drug

TT-FS - Tribulus terrestris food supplement

Reported results on the antibacterial effects of Tribulus terrestris extracts are very different and it seems that this effect is correlated with the originating geographical area of the plant and consequently with the phytochemical profile [8]. An herbal preparation used for its anabolic properties but with extra antibacterial effects could be a solution for the persons who frequently use doping agents because anabolic steroids have a substantial impact on the immune function. It is well-known that a chronic intake of anabolic steroids leads to the occurrence of an inflammatory process in the sebaceous gland, which makes the area susceptible to infections either with Staphylococcus aureus or with Propionibacterium acnes.

Urinary tract infections are part of the clinical signs of anabolic steroid use. Long term anabolic steroid use is often associated with an increase in prostatic volume, bladder irritation, urinary frequency, micturition.

The concentration of the extract obtained from the herbal drug was perhaps lower than the extract contained in the capsules. Because of the limited data on the label, it is impossible to know the exact concentration of the capsules or the solvent used for the extraction. The highest activity was against MRSA and Klebsiella pneumoniae. The polyphenols contained in Tribulus terrestris fruits could also be responsible for the antibacterial activity [24]. Before a valid conclusion could be drawn, in vivo evaluation of this antibacterial effect should be conducted because there is not always a correlation between in vitro and in vivo actions. Taking into account the bioavailability of the compounds with the alleged antibacterial action, oral administration is somewhat unlikely to lead to effective systemic concentrations.

Nevertheless, because of the significant differences between these analyzed marketed products, comparable results after intake are impossible to achieve. An increased intake of herbal drug, to reach, for example, the content of polyphenols from the capsules increases the ingestion of toxic trace elements like aluminum, which in time can cause severe disorders. There is also the risk of adulteration, a procedure very often encountered in the industry of supplements, as was shown by Radu et al. [25]. Standardization of extracts intended to be consumed as supplements and their rigorous quality control are necessary conditions before the final release on the market.

\section{Conclusions}

Aiming to analyze two highly consumed marketed products with Tribulus terrestris we conducted specific phytochemical and pharmacological determinations. Both Tribulus terrestris products exhibited strong antioxidant activity in vitro, but only the extract from the capsules had an antibacterial effect. Trace elements were quantified, and dose-related risks were outlined.

This study raises awareness regarding the safe use of herbal supplements and the importance of accurate control of these supplements with an emphasis on the possible risks assumed by inadvertent consumption which is quite high in young athletes because of their tendency to ingest higher doses 
than recommended, based on the "testosterone boosting" effect.

Acknowledgments: This research was supported by a project funded by the Internal Research Grants of the University of Medicine and Pharmacy of Târgu Mureş, Romania (grant contract for execution of research projects nr. 15609/10/29.12.2017).

\section{References}

1.CHHATRE, S., NESARI, T., KANCHAN, D., SOMANI, G., SATHAYE, S., Phytopharmacological overview of Tribulus terrestris. Pharmacogn, Rev. 8, 2014, 1545-1551.

2.ADAIKAN, P.G., GAUTHAMAN, K., PRASAD, R.N.V., History of herbal medicines with an insight on the pharmacological properties of Tribulus terrestris, Aging Male, 4, 2001, 4163-4169.

3.GAUTHAMAN, K., ADAIKAN, P.G., PRASAD, R.N.V., Aphrodisiac properties of Tribulus Terrestris extract (Protodioscin) in normal and castrated rats, Life Sci., 71, 2002, 121385-121396.

4.SINGH, S., NAIR, V., GUPTA, Y., Evaluation of the aphrodisiac activity of Tribulus terrestris Linn. in sexually sluggish male albino rats, J. Pharmacol. Pharmacother., 3, 2012, 143-148.

5.GAUTHAMAN, K., GANESAN, A.P., Sexual Effects of Puncturevine (Tribulus terrestris) Extract (Protodioscin): An Evaluation Using a Rat Model, J. Altern. Complement. Med., 9, 2003, 2257-2265.

6.GANZERA, M., BEDIR, E., KHAN, I. A., Determination of steroidal saponins in Tribulus terrestris by reversed-phase high-performance liquid chromatography and evaporative light scattering detection, J. Pharm. Sci., 90, 2001, 111752-111758.

7.DE COMBARIEU, E., FUZZATI, N., LOVATI, M., MERCALLI, E., Furostanol saponins from Tribulus terrestris, Fitoterapia, 74, 2003, 6583-6591.

8.HASHIM, S., BAKHT, T., BAHADAR MARWAT, K., JAN, A., Medicinal properties, phytochemistry and pharmacology of Tribulus terrestris L. (Zygophyllaceae), Pakistan J. Bot., 46, 2014, 1399-1404.

9.ZHELEVA-DIMITROVA, D., OBRESHKOVA, D., NEDIALKOV, P., Antioxidant activity of Tribulus terrestris - a natural product in infertility therapy, Int. J. Pharm. Pharm. Sci., 4, 2012, 45084511.

10.SINGLETON, V.L., ORTHOFER, R., LAMUELA-RAVENTOS, R.M., Analysis of total phenols and other oxidation substrates and antioxidants by means of Folin-Ciocalteu reagent, Methods Enzymol., 299, 1999, 1974152-1974178.

11.KAUR, M., HANDA, S., Physicochemical, Phytochemical Studies and Haemolytic Activity of Different Extracts of Sapindus mukorossi. Int. J. Pharm. Pharm. Res., 3, 2015, 3173-3182.

12.OLAH, N.K., PETRESCU, S., MARIAN, E., JURCA, T., MARC, F., DOBJANSCHI, L., The study of antioxidant capacity in extracts from vegetal sources with hypoglycaemic action, Rev. Chim., 70, 2019, 102-106.

13.RE, R., PELlEGRINI, N., PROTEGGENTE, A., PANNAlA, A., YANG, M., RICE-EVANS, C. Antioxidant Activity Applying an Improved ABTS Radical Cation Decolorization Assay. Free Radic. Biol. Med., 26, 1999, 91231-91237.

14.ŞTEFĂNESCU, R.E., ESIANU, S., LACZKÓ-ZÖLD, E., MARE, A., TUDOR, B., Short Period Storage Impact on Bioactive Constituents from Bilberries and Blueberries, Acta Medica Marisiensis, 63, 2017, 287-290.

15.CHEOK, C.Y., SALMAN, H.A.K., SULAIMAN, R., Extraction and quantification of saponins: A review, Food Res. Int., 26, 2014, 5916-5940.

16.GUCLU-USTUNDAG, Ö., MAZZA, G., Saponins: Properties, applications and processing. Crit. Rev. Food Sci. Nutr., 47, 2007, 47231-47258.

17.TABART, J., KEVERS, C., PINCEMAIL, J., DEFRAIGNE, J.O., DOMMES, J., Comparative antioxidant capacities of phenolic compounds measured by various tests, Food Chem., 113, 2009, 41226-41233.

18.HAMMODA, H.M., GHAZY, N.M., HARRAZ, F.M., RADWAN, M.M., Elsohly, M.A., 
ABDALLAH, I.I., Chemical constituents from Tribulus terrestris and screening of their antioxidant activity, Phytochemistry, 92, 2013, 92153-92159.

19.MALAVIGE, L., LEVY, J., Erectile dysfunction and diabetes mellitus, J. Sex. Med., 6, 2009, 61232-61247.

20.LEAL, A.S., PRADO, G., GOMES, T.C.B., SEPE, F.P., DALMÁZIO, I., Determination of metals in medicinal plants highly consumed in Brazil. Brazilian J. Pharm. Sci., 49, 2013, 3599-3607.

21.***World Health Organization, Vitamin and mineral requirements in human nutrition, Second edition, 341, 2004.

22.***E.F.S., Safety of aluminium from dietary intake - Scientific Opinion of the Panel on Food Additives, Flavourings, Processing Aids and Food Contact Materials (AFC). EFSA J., 2008, 75417534.

23.ZIRWAS, M.J., MOLENDA, M.A., Dietary Nickel as a cause of systemic contact Dermatitis, $J$. Clin. Aesthet. Dermatol., 2, 2009, 639-643.

24.WANG, B., JI, S., ZHANG, H., ZHAO, L., LV, L., LI, Y., ZHOU, G., ZHANG, G., Liquid chromatography tandem mass spectrometry in study of the pharmacokinetics of six steroidal saponins in rats, Steroids, 78, 2013, 131164-131170.

25. RADU, G.L., POPESCU, A.M., NICULAE, C.G., RADUCANU, A.E., ONISEI, T., Identification by Liquid Chromatography-Mass Spectrometry of Herbal Food Supplements Adulterated with PDE-5 Inhibitors, Rev. Chim., 66, 2015, 1-5.

$\overline{\text { Manuscript received: } 6.03 .2020}$ 